\title{
千岛湖地区常见木本植物性状和相对多度对幼苗 植食作用的影响
}

骆杨青 ${ }^{1}$ 余梅生 $^{2}$ 余晶晶 ${ }^{1}$ 郑诗璐 ${ }^{1}$ 刘佳佳 ${ }^{1}$ 于明坚 ${ }^{*}$

1浙江大学生命科学学院, 杭州 310058; 淳安县新安江开发总公司, 浙江淳安 311700

摘 要 植食性动物取食植物(植食作用)一直是生物多样性和生态系统功能研究的热点问题。植食作用能够显著影响植物幼 苗的生长, 从而决定种群更新动态。以往的研究较少讨论植物的不同性状对幼苗植食作用差异的重要性, 也较少将之与物种 相对多度对植食作用的影响进行比较。该文以千岛湖地区的16种常见木本被子植物幼苗为研究对象, 调查植物的11种功能性 状和相对多度, 并运用回归模型和方差分解方法, 研究植物功能性状和物种相对多度对幼苗叶片受昆虫植食损伤(虫食率)的 相对重要性, 探讨虫食率对植物功能性状的依赖性以及物种相对多度对虫食率的影响。结果表明叶片功能性状(即叶片碳氮 比、叶片厚度)和物种相对多度对虫食率有重要影响，二者共解释了种间虫食率变异的 $54 \%$ 。在这些性状中, 有更高的防御能 力、更低的营养成分和更高相对多度的物种虫食率更低。我们建议在未来的植食作用研究中，应考虑基于个体水平的功能性 状和基于群落水平的相对多度。

关键词 植物性状; 相对多度; 虫食率; 木本植物; 千岛湖

引用格式: 骆杨青, 余梅生, 余晶晶, 郑诗璐, 刘佳佳, 于明坚 (2017). 千岛湖地区常见木本植物性状和相对多度对幼苗植食作用的影响. 植物生态学 报, 41, 1033-1040. doi: 10.17521/cjpe.2017.0073

\section{Effects of plant traits and the relative abundance of common woody species on seedling herbivory in the Thousand Island Lake region}

\author{
LUO Yang-Qing ${ }^{1}$, YU Mei-Sheng ${ }^{2}$, YU Jing-Jing ${ }^{2}$, ZHENG Shi-Lu ${ }^{1}$, LIU Jia-Jia ${ }^{1}$, and YU Ming-Jian ${ }^{1 *}$ \\ ${ }^{1}$ College of Life Sciences, Zhejiang University, Hangzhou 310058, China; and ${ }^{2}$ Xin'an River Development Corporation, Chun'an, Zhejiang 311700, China
}

\begin{abstract}
Aims Plant-herbivore interaction is a hot topic in the study of biodiversity and ecosystem functions. Herbivores can negatively affect seedling growth and therefore can alter the dynamics of plant recruitment. However, previous studies do not fully reveal the relative importance of different plant functional traits on herbivory intensity and rarely link herbivory to the relative abundance of plant species.

Methods Here, we measured 11 plant functional traits and the relative abundance of seedlings of 16 common woody species in the subtropical forests on 29 islands in Thousand Island Lake, East China. We then used multivariate regression and variance partitioning to test the contribution of functional traits and the relative abundance to interspecific differences of insect herbivory intensity.

Important findings Our study found that both plant functional traits (e.g. carbon nitrogen ratio, leaf thickness) and the relative abundance of woody species played important roles in herbivory intensity, and they jointly contributed $54 \%$ of the variance of the interspecific differences. Among these factors, species with higher defensive ability, lower nutrient content and higher relative abundance had lower herbivory intensity. We suggest to consider both individual level traits (functional traits) and community level attributes (the relative abundance) in future herbivory studies.
\end{abstract}

Key words plant traits; relative abundance; insect herbivory; woody plant; Thousand Island Lake

Citation: Luo YQ, Yu MS, Yu JJ, Zheng SL, Liu JJ, Yu MJ (2017). Effects of plant traits and the relative abundance of common woody species on seedling herbivory in the Thousand Island Lake region. Chinese Journal of Plant Ecology, 41, 1033-1040. doi: 10.17521/cjpe.2017.0073

收稿日期Received: 2017-03-23 接受日期Accepted: 2017-08-29

* 通信作者Author for correspondence (E-mail: fishmj@zju.edu.cn) 
植食性动物对植物的植食作用(herbivory)是生 态系统中动植物之间重要的相互关系(Tilman et al., 2012), 植食活动对植物种群、群落和食物网结构都 有较大的影响(Bagchi et al., 2014), 是生物多样性和 生态系统功能研究的核心问题(曾凡勇和孙志强, 2014)。其中, 植物功能性状对植食作用的影响受到 了广泛的关注(王小菲等, 2015; 禹海金等, 2015)。

植物的叶片形态性状和化学成分一直被认为是 影响虫食率的主要因素(Awmack \& Leather, 2002; Poorter et al., 2004)。形态性状包括叶厚度、叶干物 质含量和比叶质量等, 主要影响植物的纤维素含量 和适口性(Poorter et al., 2009)。叶干物质含量和比叶 质量越高的叶片含有越高的组织密度, 通常可以防 御植食性动物从而较少受到植食侵害。化学成分表 征植物化学组成包括碳水化合物、含氮化合物和防 御性代谢产物等, 这些化合物能够直接影响植物的 营养性和防御性成分组成。碳水化合物中, 纤维素 含量影响植食性动物的消化, 高浓度可溶性糖能稀 释其他营养物质而产生对虫食的负作用(Awmack \& Leather, 2002)。含氮化合物(如氨基酸等)一般是植 食性动物的直接营养来源, 部分含氮化合物(如生 物碱)也可以防御动物取食(Carmona et al., 2011)。防 御性代谢产物(如酚类、萜类)多对植食性动物有害, 显著影响虫食率(谢辉等, 2012; Wetzel et al., 2016)。

植物的其他特征, 如相对多度等被认为可能是 影响虫食率的因素(Coley, 1980; Haukioja \& Koricheva, 2000; Farji-Brener, 2001)。一方面, 物种相对多 度通常能够代表该物种在群落中的适合度, 而植物 性状对适合度有强烈影响(Shipley et al., 2016)。植物 整个生活史阶段的若干个功能性状的共同作用在很 大程度上决定了植物的适合度。适合度高的物种通 常多度也高, 具有较高的个体生存、繁殖和生长率, 可反映为受到较低的虫食影响等(Shipley et al., 2016)。另一方面, 相对多度高的物种密度通常较高, 密度制约(negative density dependence)相关的研究 结果提示密度或多度高的植物更易受到专一性植食 动物的取食, 从而影响个体的生长生存(Comita et al., 2010)。

以往大部分研究集中在植物功能性状对植食性 动物取食的影响及其机制上, 少有研究综合植物群 落结构特征, 探讨不同植物功能性状和多度特征对 于植物种间虫食特征差异的重要性。本研究以千岛
湖岛屿上 16 种常见木本被子植物的幼苗为调研对象, 调查植物功能性状和物种相对多度等因素及幼苗叶 片受植食性动物(本研究主要为昆虫)损伤的强度, 并提出以下两个假设: (1)叶片的营养和防御性状共 同影响虫食率, 其中的碳氮比和叶干物质含量是相 对重要的性状; (2)群落中相对多度高的物种叶片受 虫食损伤低。本研究旨在进一步研究植物性状对种 间虫食率差异的相对重要性, 并探讨虫食特征能否 反映物种适合度, 为生态系统中动植物相互作用和 森林生态系统功能等研究提供资料。

\section{1 材料和方法}

\section{1 研究地区概况}

千岛湖 $\left(29.37^{\circ}-29.83^{\circ} \mathrm{N}, 118.57^{\circ}-119.25^{\circ} \mathrm{E}\right)$ 位 于浙江省西南部, 是1959年建成的新安江水电站截 新安江而成的大型人工湖泊, 水域面积约为 $573 \mathrm{~km}^{2}$ 。在最高水位海拔 $108 \mathrm{~m}$ 时, 水库拥有 1078 个 $0.25 \mathrm{hm}^{2}$ 以上的岛屿。该地区属亚热带季风气候, 年平均气温约 $17.0{ }^{\circ} \mathrm{C}, 1$ 月份最低气温 $-7.6{ }^{\circ} \mathrm{C}, 7$ 月 份最高气温 $41.8{ }^{\circ} \mathrm{C}$; 年降水量 $1430 \mathrm{~mm}$, 降雨集中 在 4 月到 6 月的雨季。本研究位于千岛湖中心湖区, 选取29个面积跨度 $0.08-1158.00 \mathrm{hm}^{2}$ 的岛屿(据最新 的GIS 计算结果), 每个岛屿的植被以次生马尾松 (Pinus massoniana) 林为主, 种类组成高度相似 $(\mathrm{Hu}$ et al., 2016)。

\section{2 数据采集}

本研究调查 16 个植物物种幼苗(胸径 $<1 \mathrm{~cm}$, 高 度50-200 cm), 以植食性昆虫造成的叶片损伤百分 比作为虫食率(图1)。16个物种包括 9 种灌木和 7 种乔 木(表1), 是该地区植物群落的主要组分。于2015年 9-10月, 在29个岛屿的样地随机选择每个岛屿每个 物种最多10株幼苗, 16个物种的幼苗在研究岛屿上 都有足够的样本。

在每株幼苗上, 随机方向上调查3根枝条, 每根 枝条带有5枚成熟的完全扩展的叶片, 总共15枚叶 片用来评估虫食率。计算由以下植食性昆虫造成的 叶面积损伤: 嚼食型、挖食型、潜食型、吸食型和 造瘱型(Schuldt et al., 2010)。根据2014年9-10月的预 试验结果，这5类叶片损伤合并计算并划分为 6 个损 伤等级记录 $(0 、 0-3 \% 、 3 \%-10 \% 、 10 \%-25 \%$ 、 $25 \%-50 \% 、 50 \%-100 \%)$ 。叶片损伤百分比=被损伤 的叶面积/总的叶面积 $\times 100 \%$ 。每个叶片的损伤等 


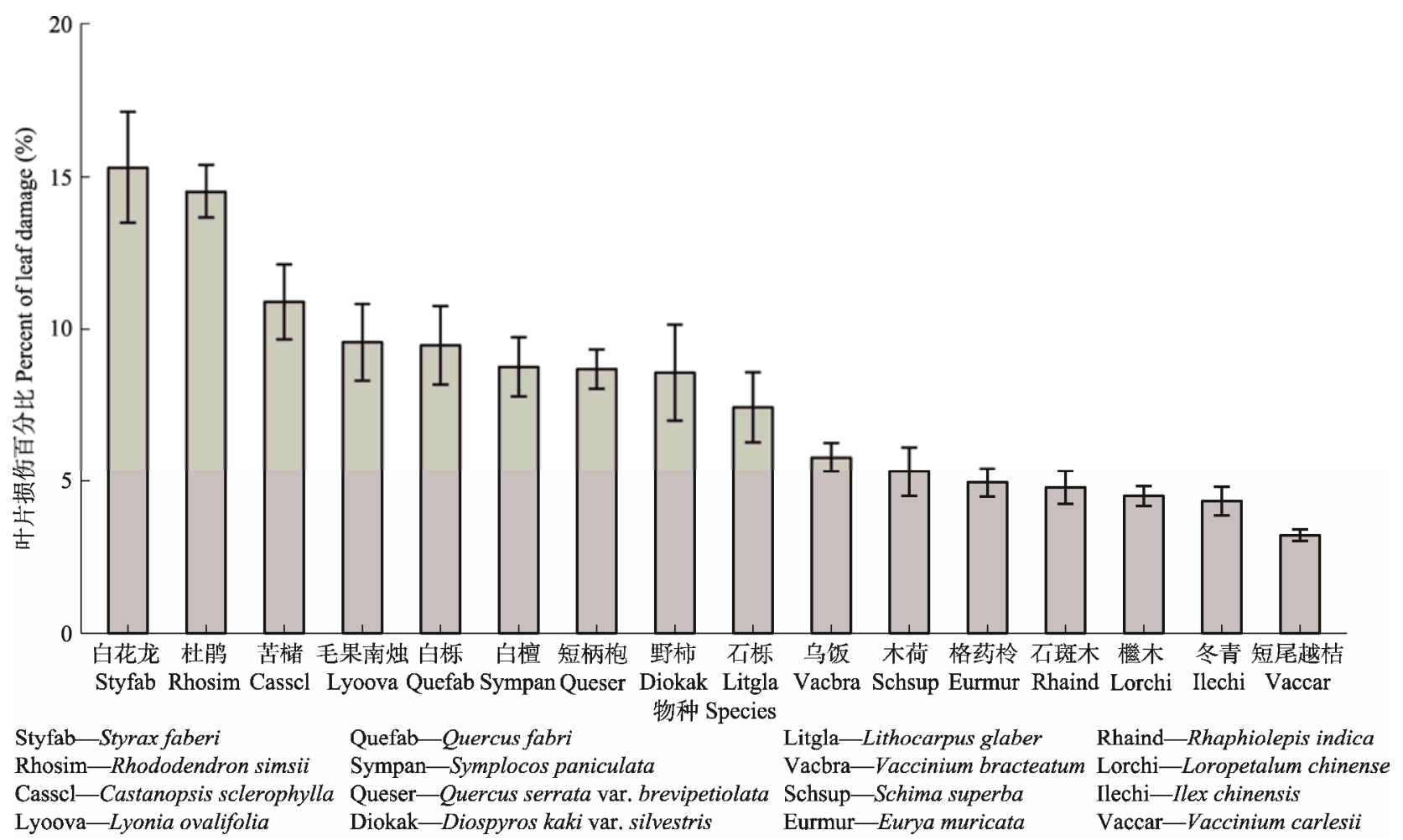

图1 16种常见木本植物幼苗受虫食损伤的平均叶片面积百分比(平均值土标准误差)。

Fig. 1 Percentages of leaf damage by insect herbivores for seedlings of 16 common woody species (mean $\pm S E$ ).

表1 选取虫食调查的16种木本植物的相关信息, 包括种名, 叶生活周期(落叶/常绿), 生长型(乔木/灌木), 光耐受性(耐阴/阳性), 分布的岛屿数, 幼苗 样本量

Table 1 Information on the 16 woody species for insect herbivory survey, including species, leaf lifespan (deciduous/evergreen), growth form (tree/shrub), light tolerance (shade tolerance/shade intolerance), number of sampling island, and number of seedling sample

\begin{tabular}{|c|c|c|c|c|c|}
\hline $\begin{array}{l}\text { 种名 } \\
\text { Species }\end{array}$ & $\begin{array}{l}\text { 叶生活周期 } \\
\text { Leaf lifespan }\end{array}$ & $\begin{array}{l}\text { 生长型 } \\
\text { Growth form }\end{array}$ & $\begin{array}{l}\text { 光耐受性 } \\
\text { Light tolerance }\end{array}$ & $\begin{array}{c}\text { 分布岛屿数 } \\
\text { No. of sampling island }\end{array}$ & $\begin{array}{c}\text { 幼苗样本量 } \\
\text { No. of seedling samples }\end{array}$ \\
\hline 野柿 Diospyros kaki var. silvestris & 落叶 Deciduous & 乔木 Tree & 耐阴 Tolerance & 8 & 32 \\
\hline 白花龙 Styrax faberi & 落叶 Deciduous & 灌木 Shrub & 耐阴 Tolerance & 9 & 55 \\
\hline 白檀 Symplocos paniculata & 落叶 Deciduous & 灌木 Shrub & 不耐阴 Intolerance & 20 & 97 \\
\hline 苦槠 Castanopsis sclerophylla & 常绿 Evergreen & 乔木 Tree & 耐阴 Tolerance & 9 & 32 \\
\hline 石栋 Lithocarpus glaber & 常绿 Evergreen & 乔木 Tree & 耐阴 Tolerance & 8 & 45 \\
\hline 冬青 Ilex chinensis & 常绿 Evergreen & 乔木 Tree & 耐阴 Tolerance & 23 & 164 \\
\hline 木荷 Schima superba & 常绿 Evergreen & 乔木 Tree & 耐阴 Tolerance & 6 & 37 \\
\hline 石斑木 Rhaphiolepis indica & 常绿 Evergreen & 灌木 Shrub & 耐阴 Tolerance & 20 & 174 \\
\hline 白栋 Quercus fabri & 落叶 Deciduous & 乔木 Tree & 不耐阴 Intolerance & 21 & 65 \\
\hline 乌饭树 Vaccinium bracteatum & 常绿 Evergreen & 灌木 Shrub & 耐阴 Tolerance & 21 & 144 \\
\hline 短柄枹 Quercus serrata var. brevipetiolata & 落叶 Deciduous & 乔木 Tree & 不耐阴 Intolerance & 20 & 112 \\
\hline 毛果南烛 Lyonia ovalifolia & 落叶 Deciduous & 灌木 Shrub & 不耐阴 Intolerance & 10 & 44 \\
\hline 格药柃 Eurya muricata & 常绿 Evergreen & 灌木 Shrub & 耐阴 Tolerance & 21 & 191 \\
\hline 杜鹃 Rhododendron simsii & 落叶 Deciduous & 灌木 Shrub & 不耐阴 Intolerance & 24 & 183 \\
\hline 聯木 Loropetalum chinense & 常绿 Evergreen & 灌木 Shrub & 耐阴 Tolerance & 26 & 240 \\
\hline 短尾越桔 Vaccinium carlesii & 常绿 Evergreen & 灌木 Shrub & 耐阴 Tolerance & 22 & 321 \\
\hline
\end{tabular}

级由人为野外实地估算进行分类并记录。每个叶片 的叶面积损伤以各个损伤等级的平均百分比 (0、 $1.5 \% 、 6.5 \% 、 17.5 \% 、 37.5 \% 、 75 \%)$ 计算，每株幼苗
的虫食率以该株幼苗上15枚叶片的平均损伤计算。 为确保虫食调查过程中估算的准确性，所有损伤面 积估算按同一分级标准进行, 并用35张标准虫食率 
的图片在每次调查前进行汶览校对, 以减小调查过 程中的误差。

2014年, 在千岛湖库区采集样本, 目标物种随 机取5-10株长势良好的成年个体, 具体方法详见文 献(余晶晶等, 2017)。在每个个体的冠层采集当年生 向阳面的20枚完整无病斑的成熟叶片，测定如下4 个变量来表征植物叶形态性状: 叶厚度、叶面积、 叶干物质含量和比叶质量。叶干物质含量表示叶片 干质量和叶片鲜质量的比值。比叶质量表示叶片干 质量和新鲜叶片的单面面积的比值。还测定了植物 的叶片化学性状: 碳含量、氮含量和碳氮比。测量 方法为每个物种测量 5 株个体的碳氮含量, 每株个 体取10片干燥叶片, 粉碎过 100 目篮, $80{ }^{\circ} \mathrm{C}$ 烘干至 恒质量, 称取 $50 \mathrm{mg}$ 待测样品, 用锡箔纸包裹, 元素 分析仪碳氮模式分析测试, 最后对每个物种 5 株个 体的碳氮含量取平均值。此外, 还参考《浙江植物 志》和《中国植物志》记录植物物种水平的其他功 能性状: 叶生活周期(常绿/落叶)、生长型(乔木/灌 木)、光耐受性(耐阴/不耐阴)和最大树高。

2014-2015年, 采用CTFS森林动态样地的标准 方法对 29 个岛屿的样地内胸径 $1 \mathrm{~cm}$ 以上的木本植物 个体进行复查, 对2009-2010年初次调查中已挂牌 的个体重新调查胸径、树高, 对新增个体进行标记 和挂牌, 并记录其胸径和树高, 具体调查方法及结 果详见文献(Hu et al., 2016)。本研究使用这次复查 中各物种的相对多度值, 表示各物种的多度与总的 多度的比值。

\section{3 数据分析}

本研究采用多元线性回归模型分析植物功能性 状和物种相对多度等变量对虫食率的影响。首先, 对所有连续型自变量以2倍自变量的标准差作标准 化, 保证变量尺度的可比性(Gelman, 2008), 并对各 物种叶片虫食百分比取平均值, 并作逻辑斯蒂转换 以保证正态性(Warton \& Hui, 2011)。然后, 对变量 进行共线性检验, 发现叶干物质含量/叶厚度与比 叶质量高度相关(Pearson相关系数 $|r|>0.60$ ), 由于 叶干物质含量对植食作用影响较为显著(Schuldt et al., 2012), 保留叶干物质含量。而另一组高度相关 变量碳氮比/叶面积和氮含量, 碳氮比在后续模型 中相对氮含量有更低的赤池信息量准则(AICc)值 $($ AICc $=-5.13$ vs. AICc $=-0.86)$, 保留碳氮比。最后 得到包括7个变量的模型: 叶厚度、叶面积、叶干物
质含量、碳含量、碳氮比、最大树高和相对多度。

对上述模型进行模型选择, 由于最优模型之间 具有相近的AICc值 $(\triangle \mathrm{AICc}<2)$, 对赤池重量(Akaike weight, $\omega_{+}$)累积 $95 \%$ 以上的模型组内的变量计 算其相对重要性, 䇥选出重要的变量得出最佳模型 (Burnham \& Anderson, 2003), 并对模型的统计假设 进行检验。同时，对分类型变量与虫食率做线性回 归, 观察对虫食特征的影响。此外, 对最佳模型使用 方差分解, 以解释模型中各个变量对 16 个物种的平 均虫食的独立和联合作用。以上所有数据分析和图 形输出都基于R 3.3.2 (R Core Team, 2016)完成。

\section{2 结果}

本研究共调查了 16 个常见木本植物共计 1936 株幼苗(共有28 884枚叶片)上的虫食特征情况。各物 种平均植食性昆虫损伤叶片面积百分比从 $3.2 \%$ (短 尾越桔)到 $15.3 \%$ (白花龙), 总体平均为 $7.9 \%$ (图1)。 其中, 虫食率最高的两个物种白花龙(15.3\%)和杜鹃 (14.5\%)的叶片碳氮比相对较低, 在群落中相对多度 较低, 都是落叶物种; 而虫食率最低的 3 个物种短 尾越桔(3.2\%)、冬青(4.3\%)和聯木(4.5\%)叶片碳氮比 相对较高, 叶片较厚, 是群落中优势常绿物种。

根据最佳模型结果, 在 $95 \%$ 置信区间内, 碳氮 比、叶厚度、相对多度是模型中极为重要的 3 个变量 $\left(\omega_{+}=0.83 ; 0.60 ; 0.53\right.$, 图2), 相对重要性大于其他 变量 $\left(0.08 \leqslant \omega_{+} \leqslant 0.12\right)$ 。因此, 包括碳氮比(回归系 数 $(E s t i)=-0.22 ; p=0.01)$ 、叶厚度 $(E s t i=-0.17 ; p=$ $0.03)$ 和相对多度 $(E s t i=-0.18 ; p=0.05)$ 的 3 个变量的 预测模型是最佳模型(adjusted R squared $=0.54 ; p<$ 0.01)。同时，根据最佳模型的偏残差图(图3), 验证 巩固了该模型的线性, 并直观反映了碳氮比、叶厚 度和相对多度对虫食率的影响。最佳模型中的3个自 变量均与叶片损伤百分比显著相关, 该物种叶片中 的碳氮比越高, 叶厚度越大, 在群落中的相对多度 越高, 该物种由虫食引起的叶片损伤越小。其他变 量中, 比叶质量和氮含量在与叶片损伤的线性回归 中表现显著的相关性 $(E s t i=-0.23, p=0.03 ; E s t i=$ $0.25, p=0.04)$ 。而生长型和光耐受性在与叶片损伤 的线性回归中未表现出显著的相关性 $(p>0.05)$ 。叶 生活周期与碳氮比、叶厚度都高度相关, 在与叶片 损伤的线性回归中表现出极显著的相关性(Esti = $-0.31, p<0.001)$, 常绿物种的叶片损伤百分比远小 


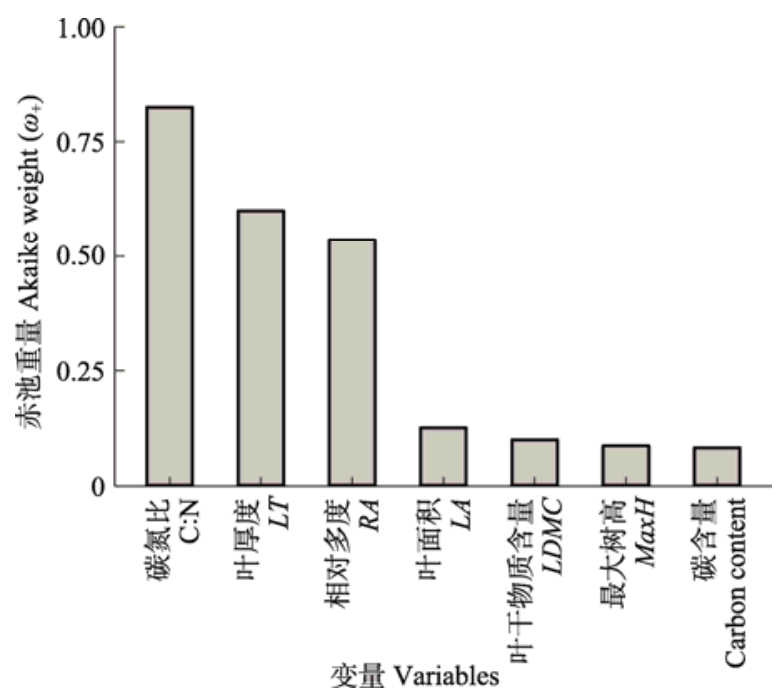

图2 模型平均计算中7个变量的相对重要性对比, 估计为 在 $95 \%$ 置信集中的赤池重量 $\left(\omega_{+}\right)$之和。

Fig. 2 The relative importance of seven variables measured as the sum of Akaike weight $\left(\omega_{+}\right)$in $95 \%$ confidence set with model averaging. $\mathrm{C}: \mathrm{N}$, carbon nitrogen ratio; $L A$, leaf area; $L D M C$, leaf dry matter content; LT, leaf thickness; MaxH, maximum tree height; $R A$, relative abundance.

于落叶物种。

在方差分解中, 碳氮比、叶厚度和相对多度 3 个 变量共解释了 $54 \%$ 的叶片损伤的方差变异。其中, 碳氮比独立解释了 $28 \%$ 的方差, 对叶片损伤程度具 有更大的影响。而叶厚度和相对多度分别独立解释 了16\%和14\%的方差。3个变量相对独立, 共同解释 部分较小, 不能被解释的部分占 $46 \%$ 。

\section{3 讨论}

本研究通过结合 16 个常见木本植物的功能性状
和群落结构特征，为探讨物种之间虫食特征差异的 影响因素提供了一个新的视角。研究结果表明: (1) 碳氮比和叶厚度等性状与虫食率有明显的负相关关 系; (2)叶生活周期长的植物叶片上的虫食率相对较 低; (3)物种相对多度对种间虫食率差异有较强影响。

本研究发现, 碳氮比是影响植物种间虫食率差 异的最显著的变量(图3)。碳氮比与虫食率表现出显 著的负相关关系, 碳氮比越高的物种遭受的虫食越 低。植物叶片中的碳水化合物, 如纤维、糖类和固醇 类, 主要作为防御性成分对虫食率有消极影响, 高 密度的纤维素、高浓度的糖类常被作为植物防御手 段降低昆虫侵害(Awmack \& Leather, 2002)。而含氮 化合物如氨基酸, 通常表征蛋白质含量, 在大部分 植物中作为营养性成分吸引昆虫取食(Poorter et al., 2004; Joern et al., 2012)。因此, 碳氮比作为植物的防 御和营养性状能够对虫食率产生影响(Loranger et al., 2012)。植物中越高的碳氮比表明叶片中存在的防御 物质含量越高、营养物质含量越少, 从而影响昆虫取 食选择并导致较低的虫食率(Mithofer \& Boland, 2012), 本研究结果与之一致。而氮含量与碳氮比高 度相关, 也表现出与叶片损伤强度的显著相关性, 表明营养性状在种间虫食率差异中的重要影响。此 外, 叶厚度与虫食率也表现出显著的负相关关系, 越厚的叶片受到的虫食损伤越小。本研究中叶厚度 与比叶质量高度相关 $(r=0.67, p=0.005)$, 通常较高 的叶厚度和比叶质量代表较高的叶片组织密度, 导 致叶片营养物质含量和适口性的降低。因此, 叶厚
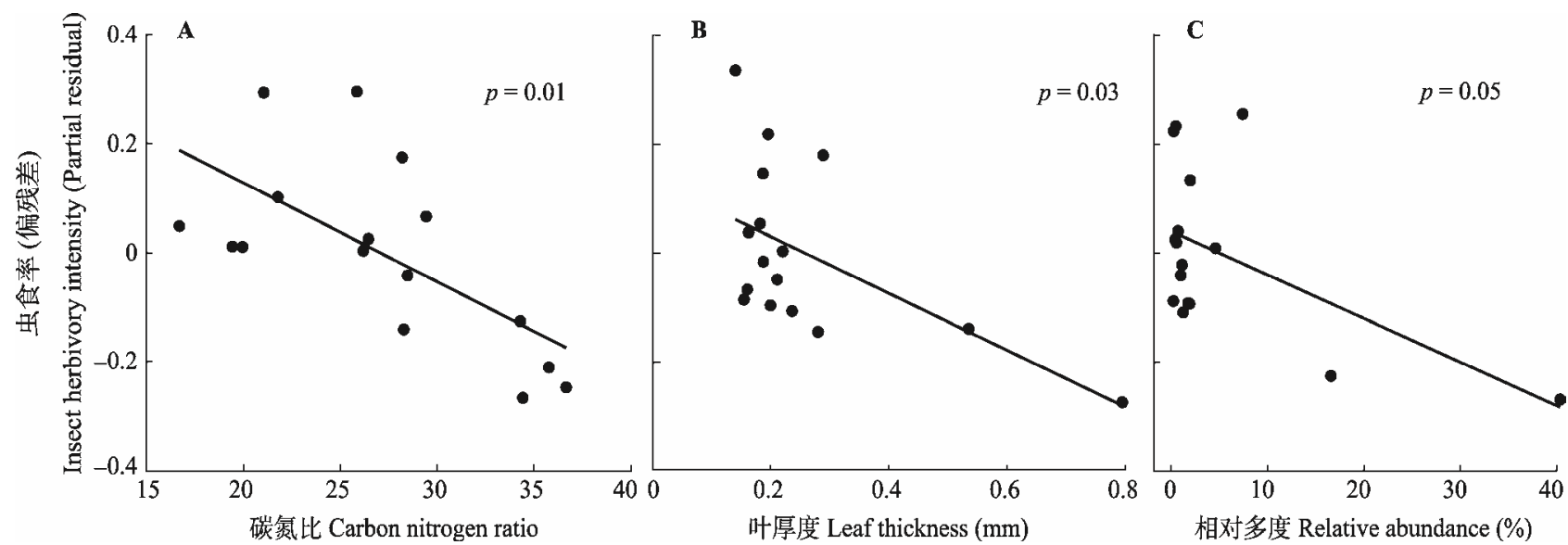

图3 最佳模型中3个变量对于千岛湖16种木本植物幼苗的平均叶片虫食损伤百分比(排除了其他变量影响的偏残差)的独立影 响。A, 碳氮比。B, 叶厚度。C, 相对多度。

Fig. 3 Independent effects of three variables in the best model on mean percentage of leaf damage (partial residuals excluding the effects of other variables) on seedlings of 16 woody species in the Thousand Island Lake. A, Carbon nitrogen ratio. B, Leaf thickness. C, Relative abundance. 
度、比叶质量和叶干物质含量等结构性状在以往大 量的研究中被认为与叶片虫食率有持续的负相关关 系, 是研究虫食特征的比较稳定的性状(Cingolani et al., 2005; Poorter et al., 2009)。本研究中, 叶厚度和比 叶质量作为物理防御性状对虫食率的影响与以往研 究结果(Hanley et al., 2007)一致。而叶干物质含量的 作用不显著, 在一些种间虫食特征差异的研究中也 有类似结果。虽然叶干物质含量与虫食率的负相关 关系在单一物种内极为普遍稳定, 但是在种间关系 上, 其作用有可能被个别物种的防御性代谢产物或 其他防御手段所弱化(Elger \& Willby, 2003)。结果表 明, 与假设(1)相近, 碳氮比和叶厚度等营养和防御 性状共同影响植食性昆虫取食选择, 是影响植物种 间虫食率差异的主要因素, 但叶干物质含量对种间 虫食率差异的影响不显著。

叶生活周期与碳氮比和叶厚度都有较高的相关 性(没有包括在最佳模型中), 在与虫食率的线性回归 中显示了极显著的影响。根据成本效益理论, 生活在 林荫下的幼苗由于光照少而获得的资源有限, 而叶 生活周期长的幼苗往往有更好的防御手段(如高叶片 厚度和组织密度) 和更低的营养物质含量, 可以有效 地降低虫食带来的损伤并提高存活率(Chabot \& Hicks, 1982; Kitajima et al., 2013; Silva et al., 2015)。 本研究结果验证了叶生活周期更长的植物有更高的 碳氮比和叶厚度, 有更低的虫食损伤, 同时相关性 分析发现叶生活周期与氮含量等相关性显著, 表明 常绿阔叶植物通过提高相关的防御能力、降低营养 含量以降低虫食率, 这一结果支持最新的研究结论 (Zhang et al., 2017)。

根据相关性分析和模型结果, 相对多度越高的 物种, 其幼苗被虫食损伤的强度越小, 与假设(2)一 致。植物性状能够显著影响适合度, 而相对多度可以 作为该物种适合度的定量替代(Shipley et al., 2016)。 本研究中相对多度与其他形态、化学性状没有显著 相关性, 除上述性状外还有其他植物性状影响虫食 特征, 能够解释种间虫食率变异的 $14 \%$ 。这与密度制 约的理论不符, 但是本研究未对植食性昆虫进行调 查, 仅在虫食调查过程中观察昆虫种类, 观察到的 多为鳞翅目蛾类幼虫等寡食性昆虫, 这可能是违背 假设的一个原因(Schuldt et al., 2010); 也有研究指出, 密度制约对常见物种的作用比对稀有物种的作用弱 (Johnson et al., 2012)。因此, 在千岛湖地区相对多度
较高的物种适合度高, 密度制约的作用较小, 受虫 食损伤也较低。

综上所述, 本研究结果表明, 叶片碳氮比和叶 厚度等作为营养和防御性状在千岛湖地区共同影响 植食性昆虫的取食选择, 而相对多度高、适合度高的 物种受虫食损伤低。在模型中, 碳氮比、叶厚度和相 对多度所代表的其他性状能解释本研究地区16个植 物物种平均叶片虫食率变异的 $54 \%$ 。而其他性状如叶 面积、叶干物质含量和光耐受性等对虫食率没有表 现出明显的影响(Moles \& Westoby, 2000; Kitajima \& Poorter, 2010; Salgado-Luarte \& Gianoli, 2010)。这些 性状对虫食特征的独立及联合影响还需要进一步结 合植物群落结构特征, 对植物物种种内和种间各项 性状以及植食性昆虫种类的调查来进行分析。本研 究探讨在以往研究中较少衡量的植物功能性状和相 对多度在植物种间叶片虫食率差异中的相对重要性, 表明叶片营养和防御性状与相对多度在植食性昆虫 取食过程中的重要作用, 对促进动植物相互作用研 究有重要意义。

基金项目 国家自然科学基金(31210103908、 31361123001和31500382)。

致谢浙江淳安新安江开发总公司对项目实施提供 支持, 浙江大学南歌、巫东豪、戎福仁、秦雪妍、 邬劼和当地村民在野外调查中给予帮助, 在此一并 表示感谢。

\section{参考文献}

Awmack CS, Leather SR (2002). Host plant quality and fecundity in herbivorous insects. Annual Review of Entomology, 47, 817-844.

Bagchi R, Gallery RE, Gripenberg S, Gurr SJ, Narayan L, Addis CE, Freckleton RP, Lewis OT (2014). Pathogens and insect herbivores drive rainforest plant diversity and composition. Nature, 506(7486), 85-88.

Burnham KP, Anderson DR (2003). Model Selection and Multimodel Inference: A Practical Information-Theoretic Approach. Springer-Verlag, New York.

Carmona D, Lajeunesse MJ, Johnson MT (2011). Plant traits that predict resistance to herbivores. Functional Ecology, 25, 358-367.

Chabot BF, Hicks DJ (1982). The ecology of leaf life spans. Annual Review of Ecology and Systematics, 13, 229-259.

Cingolani AM, Posse G, Collantes MB (2005). Plant functional traits, herbivore selectivity and response to sheep grazing in Patagonian steppe grasslands. Journal of Applied

www.plant-ecology.com 
Ecology, 42, 50-59.

Coley PD (1980). Effects of leaf age and plant life history patterns on herbivory. Nature, 284, 545-546.

Comita LS, Muller-Landau HC, Aguilar S, Hubbell SP (2010). Asymmetric density dependence shapes species abundances in a tropical tree community. Science, 329, 330-332.

Elger A, Willby NJ (2003). Leaf dry matter content as an integrative expression of plant palatability: The case of freshwater macrophytes. Functional Ecology, 17, 58-65.

Farji-Brener AG (2001). Why are leaf-cutting ants more common in early secondary forests than in old-growth tropical forests? An evaluation of the palatable forage hypothesis. Oikos, 92, 169-177.

Gelman A (2008). Scaling regression inputs by dividing by two standard deviations. Statistics in Medicine, 27, 2865-2873.

Hanley ME, Lamont BB, Fairbanks MM, Rafferty CM (2007). Plant structural traits and their role in anti-herbivore defence. Perspectives in Plant Ecology, Evolution and Systematics, 8(4), 157-178.

Haukioja E, Koricheva J (2000). Tolerance to herbivory in woody vs. herbaceous plants. Evolutionary Ecology, 14, 551-562.

Hu G, Feeley KJ, Yu M (2016). Habitat fragmentation drives plant community assembly processes across life stages. PLOS ONE, 11, e0159572. doi: 10.1371/journal.pone.0159572.

Joern A, Provin T, Behmer ST (2012). Not just the usual suspects: Insect herbivore populations and communities are associated with multiple plant nutrients. Ecology, 93, 1002-1015.

Johnson DJ, Beaulieu WT, Bever JD, Clay K (2012). Conspecific negative density dependence and forest diversity. Science, 336, 904-907.

Kitajima K, Cordero RA, Wright SJ (2013). Leaf life span spectrum of tropical woody seedlings: Effects of light and ontogeny and consequences for survival. Annals of Botany, 112, 685-699.

Kitajima K, Poorter L (2010). Tissue-level leaf toughness, but not lamina thickness, predicts sapling leaf lifespan and shade tolerance of tropical tree species. New Phytologist, 186, 708-721.

Loranger J, Meyer ST, Shipley B, Kattge J, Loranger H, Roscher C, Weisser WW (2012). Predicting invertebrate herbivory from plant traits: Evidence from 51 grassland species in experimental monocultures. Ecology, 93, 2674-2682.

Mithofer A, Boland W (2012). Plant defense against herbivores: Chemical aspects. Annual Review of Plant Biology, 63, 431-450.

Moles AT, Westoby M (2000). Do small leaves expand faster than large leaves, and do shorter expansion times reduce herbivore damage? Oikos, 90, 517-524.

Poorter H, Niinemets Ü, Poorter L, Wright IJ, Villar R (2009).
Causes and consequences of variation in leaf mass per area (LMA): A meta-analysis. New Phytologist, 182, 565-588.

Poorter L, van de Plassche M, Willems S, Boot RG (2004). Leaf traits and herbivory rates of tropical tree species differing in successional status. Plant Biology (Stuttg), 6, 746-754.

R Core Team (2016). R: A language and environment for statistical computing. https://www.r-project.org. Cited 2017-05-22.

Salgado-Luarte C, Gianoli E (2010). Herbivory on temperate rainforest seedlings in sun and shade: Resistance, tolerance and habitat distribution. PLOS ONE, 5, e11460. doi: 10.1371/journal.pone.0011460.

Schuldt A, Baruffol M, Böhnke M, Bruelheide H, Härdtle W, Lang AC, Nadrowski K, von Oheimb G, Voigt W, Zhou H (2010). Tree diversity promotes insect herbivory in subtropical forests of south-east China. Journal of Ecology, 98, 917-926.

Schuldt A, Bruelheide H, Durka W, Eichenberg D, Fischer M, Krober W, Hardtle W, Ma K, Michalski SG, Palm WU, Schmid B, Welk E, Zhou H, Assmann T (2012). Plant traits affecting herbivory on tree recruits in highly diverse subtropical forests. Ecology Letter, 15, 732-739.

Shipley B, de Bello F, Cornelissen JH, Laliberte E, Laughlin DC, Reich PB (2016). Reinforcing loose foundation stones in trait-based plant ecology. Oecologia, 180, 923-931.

Silva JO, Espírito-Santo MM, Morais HC (2015). Leaf traits and herbivory on deciduous and evergreen trees in a tropical dry forest. Basic and Applied Ecology, 16, 210-219.

Tilman D, Reich PB, Isbell F (2012). Biodiversity impacts ecosystem productivity as much as resources, disturbance, or herbivory. Proceedings of the National Academy of Sciences of the United States of America, 109, 10394-10397.

Wang XF, Gao WQ, Liu JF, Ni YY, Jiang ZP (2015). Plant defensive strategies and environment-driven mechanisms. Chinese Journal of Ecology, 34, 3542-3552. (in Chinese with English abstract) [王小菲, 高文强, 刘建锋, 倪妍 妍, 江泽平 (2015). 植物防御策略及其环境驱动机制. 生态学杂志, 34, 3542-3552.]

Warton DI, Hui FK (2011). The arcsine is asinine: The analysis of proportions in ecology. Ecology, 92, 3-10.

Wetzel WC, Kharouba HM, Robinson M, Holyoak M, Karban $\mathrm{R}$ (2016). Variability in plant nutrients reduces insect herbivore performance. Nature, 539, 425-427.

Xie H, Wang Y, Liu YQ, Chen LP (2012). The influence of plant constitutive defense system on phytophagous insects. Plant Protection, 38(1), 1-5. (in Chinese with English abstract) [谢辉, 王燕, 刘银泉, 陈利萍 (2012). 植物组 成型防御对植食性昆虫的影响. 植物保护, 38(1), 1-5.]

Yu HX, Ye WF, Sun MQ, Xu N, Lou SZ, Ran JX, Lou YG (2015). Three levels of defense and anti-defense responses between host plants and herbivorous insects. Chinses 
Journal of Ecology, 34, 256-262. (in Chinese with English abstract) [禹海金金, 叶文丰, 孙民琴, 徐宁, 娄少之, 由 俊祥, 娄永根 (2015). 植物与植食性昆虫防御与反防御 的三个层次. 生态学杂志, 34, 256-262.]

Yu JJ, Jin Y, Zheng SL, Hu G, Liu JL, Yuan JF, Liu JJ, Yu MJ (2017). Differentiation in leaf and branch traits of angiosperms and their relationships with species abundance in the Thousand Island Lake region. Journal of Zhejiang University (Science Edition), 44, 437-445. (in Chinese with English abstract) [余晶晶, 金毅, 郑诗璐, 胡广, 刘 金亮, 袁金风, 刘佳佳, 于明坚 (2017). 千岛湖被子植 物枝叶性状分化及其与种多度关系. 浙江大学学报(理
学版), 44, 437-445.]

Zeng FY, Sun ZQ (2014). Mechanism, hypothesis and evidence of herbivorous insect-host interactions in forest ecosystem. Acta Ecologica Sinica, 34, 1061-1071. (in Chinese with English abstract) [曾凡勇, 孙志强 (2014). 森林生态系 统中植食性昆虫与寄主的互作机制、假说与证据. 生态 学报, 34, 1061-1071.]

Zhang S, Zhang Y, Ma K (2017). The association of leaf lifespan and background insect herbivory at the interspecific level. Ecology, 98, 425-432.

责任编委: 孙书存 责任编辑: 王 葳

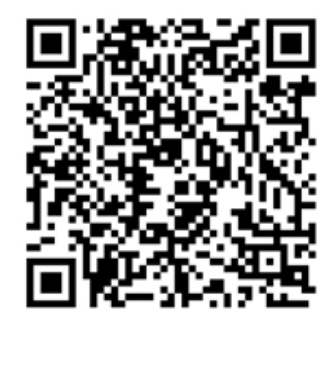

